\title{
Payload Capabilities and Operational Limits of Eversion Robots
}

\author{
Hareesh Godaba ${ }^{1,3}$, Fabrizio Putzu ${ }^{2,3}$, Taqi Abrar ${ }^{1,3}$, Jelizaveta Konstantinova ${ }^{1,3}$ and \\ Kaspar Althoefer ${ }^{1,2,3}$ \\ ${ }^{1}$ School of Electronic Engineering and Computer Science, Queen Mary University of London \\ ${ }^{2}$ School of Engineering and Materials Science, Queen Mary University of London \\ ${ }^{3}$ Centre for Advanced Robotics @ Queen Mary, Queen Mary University of London \\ h.godaba@gmul.ac.uk
}

\begin{abstract}
Recent progress in soft robotics has seen new types of actuation mechanisms based on apical extension which allows robots to grow to unprecedented lengths. Eversion robots are a type of robots based on the principle of apical extension offering excellent maneuverability and ease of control allowing users to conduct tasks from a distance. Mechanical modelling of these robotic structures is very important for understanding their operational capabilities. In this paper, we model the eversion robot as a thin-walled cylindrical beam inflated with air pressure, using Timoshenko beam theory considering rotational and shear effects. We examine the various failure modes of the eversion robots such as yielding, buckling instability and lateral collapse, and study the payloads and operational limits of these robots in axial and lateral loading conditions. Surface maps showing the operational boundaries for different combinations of the geometrical parameters are presented. This work provides insights into the design of eversion robots and can pave the way towards eversion robots with high payload capabilities that can act from long distances.
\end{abstract}

Keywords: Eversion robots, Soft robots, Inflated beams, Timoshenko beam theory, Failure modes, Operational limits, Design parameters.

\section{Introduction}

Recent years have seen huge strides in the field of soft robotics with a variety of actuation mechanisms improving the maneuverability, range of actuation and offer novel functionalities. Soft actuation mechanisms are based on different principles such as electroactuation [1, 2], shape memory effect [3], swelling of gels [4], tendon based actuation [5], pneumatic [6] and hydraulic actuation [7]. Pneumatic actuation has been very popular due to its ease of fabrication, low cost and inherent compliance of the materials. Earlier works predominantly utilized hyperelastic materials molded into soft structures and then inflated with air to cause deformation in the soft robotic bodies. However, further developments have seen the introduction of new materials, such as paper, plastics and fabrics [8-11]. In contrast to hyperelastic materials which harness large extensions in their bodies to generate actuation, these elastic materials utilise 
topological changes such as bending, folding or unfolding to generate actuation. This principle has been used to develop fabric based actuators that can exhibit linear elongations and bending, pouch type actuators that can contract and robots that can grow in length through eversion $[8,9,12]$.

Hawkes et al. utilized a polyethylene sheet to develop an apically extending soft robot that grows by everting material from its tip [12]. In this eversion robot, a long tubular sock of polyethylene was prefabricated, and one end of the tube was sealed. The sealed end was folded inwards and passed back through the center of the tube and fed into a spool. When pressure is exerted through the open end of the tube, the pressure pushes the material out of the tip and the robot grows in an everting fashion. The eversion robots have been demonstrated to be steerable and can extend at rates of up to $10 \mathrm{~m} / \mathrm{s}$ to tens of meters in length. Different applications such as surveillance and monitoring, reconfigurable antennas, a soft burrowing device, soft artificial muscles and manipulators for minimally invasive surgery have been demonstrated using the eversion principle [12-15]. Furthermore, by embedding sensing technologies, bioinspired control techniques can be implemented to control eversion robots and track complex trajectories due to their high maneuverability [16].

However, there has been minimal work on developing and characterizing eversion robots that can deliver payloads. Putzu et al. developed a pneumatic actuator based on the eversion principle in which a payload of $3 \mathrm{~N}$ was suspended using a tendon attached to the sealed end of the membrane [15]. With the large range of actuation and interesting maneuvering capabilities of eversion robots, the subsequent goals would be to equip the eversion-based robots with sensing and manipulation capabilities by attaching sensors and effectors to the tip of the robot. This requires the eversion robots to have sufficient payload capabilities to carry these components and execute manipulation tasks. The slender form factor of the eversion robots presents us with challenges such as low flexural rigidity and susceptibility to instabilities. Towards the goal of developing payload capabilities in eversion robots, we conduct here a modelling study to investigate the achievable forces under different loading conditions. We make use of analytical models of inflatable beams based on the Timoshenko beam theory to find the different failure modes and operation limits of eversion robots. 3D maps showing the operational limits and modes of failure for axial and lateral loading conditions are presented. This work lays a foundation for more exciting applications of eversion robots with high payload capabilities.

The rest of the paper is organized as follows. In section 2, we develop the analytical model for an eversion robot and describe the stresses in the robot. In section 3, we discuss the various failure modes of the eversion robots and present 3D surface maps showing the operational capabilities of the eversion robot subject to different failure modes.

\section{$2 \quad$ Analytical modelling}

An eversion robot is made of a thin elastic membrane preformed into a long tubular sock. The tubular sock is folded inwards and housed inside the robot body. When a 
pressure is applied through the open end, the material folded inwards begins to evert from the tip increasing the length of the eversion robot. A tendon is attached to the tip of the tubular sock and can be utilized to exert forces on the tip. Tension can be applied to the tendon to exert larger pressure to the eversion robot and increase its stiffness. Fig.1 shows a schematic of the eversion robot. The thickness of the membrane is denoted by $\mathbf{t}$. It is generally much smaller compared to the radius of the robot. This is to ensure a low flexural stiffness of the membrane so that it can attain high curvatures and facilitate eversion. In this paper, we model the eversion robot as an inflated beam made of a thin elastic membrane. The proximal end of the eversion robot is fixed, and a pressure denoted by $P$ is applied through this opening. The force exerted by a tendon (attached to the distal, sealed end of our eversion robot), denoted by $F_{\text {tendon }}$, and the external force, $F_{\text {ext }}$, act on the sealed end of the beam, as shown in Fig. 1. We consider the quasi static equilibrium in the eversion robot of a given radius to calculate the forces exerted by the robot at different pressures and everted lengths. Since, the thickness of the membrane is much smaller than the radius of the eversion robot and the stresses are assumed to not vary along the thickness direction of the membrane. The lengths of the robot considered are much larger as compared to the radii of the robot so that small deformation approximations can be applied.

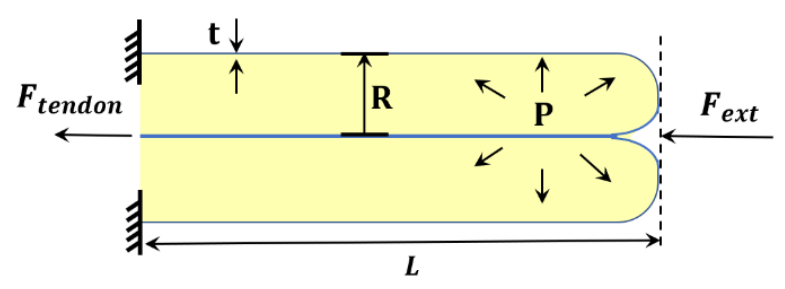

Fig. 1. Free body diagram of eversion actuator. An eversion robot of radius, $R$, wall thickness, $t$ and everted length, $\mathrm{L}$ is fixed at one end. It is subject to an internal pressure, $\mathrm{P}$, external force, $F_{\text {ext }}$ and a force, $F_{\text {tendon }}$, is exerted by the tendon at the tip.

\subsection{Force equilibrium}

When the robot is pressurized and the tip is unobstructed, the robot grows while everting. The rate of growth is given by the following equation $[17,18]$ :

$$
r=\varphi(P-Y)^{n}
$$

where $\mathrm{P}$ and $\mathrm{Y}$ are the internal pressure and the threshold pressure required for eversion, $\varphi$ is the extensibility of the system and $n$ is a power term that relates the degree of change of rate of extension to internal pressure.

When the robot is in a static equilibrium with respect to external forces, the force analysis simplifies to a static force equilibrium problem for the system. Fig. 1 shows the schematic of an eversion robot showing the forces acting on the robot in the case of equilibrium. The external force, $F_{\text {ext }}$ is balanced by the force pulling on the tendon, 
$F_{\text {tendon }}$ and the internal pressure acting on the robot body. Assuming that the cross section of the tip is circular with an effective radius, $R$, the force balance is given by:

$$
P\left(\pi R^{2}\right)=F_{\text {ext }}+F_{\text {tendon }}
$$

As we can see from the equation above, the maximum external force (or payload) is achievable when the force in the tendon is zero, i.e., the tendon is slack. So, from this point, we consider $F_{\text {tendon }}=0$, in our analysis concerning the maximum achievable forces.

\subsection{Longitudinal and circumferential stresses}

In an eversion robot, the membrane forming the robot body should be very thin and flexible in order to allow the tip to evert. Typically, the thickness of the membrane to the radius ratio is extremely small. Due to this, the membrane does not experience radial stresses when pressurized and only experiences longitudinal (or axial) and circumferential (or hoop's) stresses [19]. These stresses are denoted by $\sigma_{L}$ and $\sigma_{\theta}$ respectively. Fig. 2 shows the longitudinal and axial cross sections of an eversion robot with an everted length, $L$, showing the longitudinal and circumferential stresses.

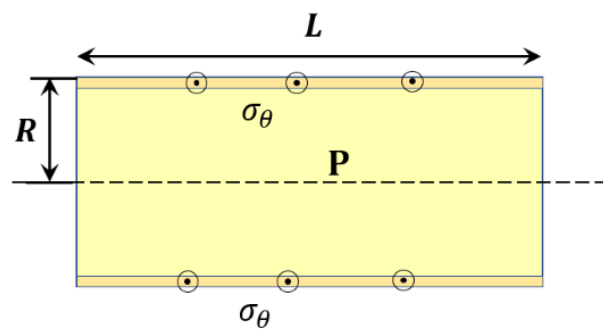

Longitudinal cross-section

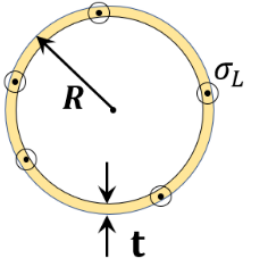

Lateral cross-section

Fig. 2. Longitudinal and lateral cross-sections showing the circumferential stress, $\sigma_{\theta}$ and longitudinal stress, $\sigma_{L}$, respectively.

In the longitudinal direction, the forces exerted by the internal pressure on the tip of the eversion robot are balanced by the longitudinal stresses in the robot body. When the thickness of the membrane is much smaller than the radius of the eversion robot, the force equilibrium in the longitudinal direction is given by:

$$
\sigma_{L}(2 \pi R t)=P\left(\pi R^{2}\right)
$$

and the longitudinal stress in the body is given by

$$
\sigma_{L}=P R / 2 t
$$

The robot everts from a fixed end that is annular in cross-section. Close to the fixed boundary, there may be boundary inhomogeneities. However, away from the fixed boundary, we consider that the circumferential stresses are uniformly distributed along 
the length of the eversion robot. Along a longitudinal cross section, the force balance is given by:

$$
\sigma_{\theta}(2 L t)=P(R L)
$$

The circumferential or hoop's stress is given by:

$$
\sigma_{\theta}=\frac{P R}{2 t}
$$

The expressions for the stresses in the membrane are similar to those of a thin-walled cylinder subject to internal pressure. The circumferential and longitudinal stresses should always be non-negative for the robot to be in operation.

\section{Results}

In this section, we discuss the various failure modes of the eversion based. The payload capability of the eversion-based robot depends on various factors such as the type of loading, the geometric parameters such as the radius, thickness of the membrane and the everted length, as well as the material properties. In this work, we consider the payload capabilities in the longitudinal and lateral loading of the eversion robot. These quantities indicate the amount of forces that the robot can deliver while operating in a given state. In the axial loading, we are mainly concerned about two main modes of failure: (1) yielding and (2) buckling due to compression. In the case of lateral loading, we consider the lateral collapse of the robot due to loss of tension along the longitudinal direction.

In this simulation, we consider low density polyethylene (LDPE) as the material for the eversion-based robot. This has been chosen as the subject material because of its use in previous works and also due to its easy processability and fabrication. The various material and geometric parameters used in the simulation are shown in Table 1 [20].

Table 1. Material and geometric parameters of the eversion robot for modelling.

\begin{tabular}{llll}
\hline Parameter & Symbol & Value & Unit \\
\hline Young's modulus & $\mathrm{E}$ & 110 & $\mathrm{MPa}$ \\
Thickness & $\mathrm{t}$ & 100 & $\mu \mathrm{m}$ \\
Yield stress & $\sigma_{y}$ & 40 & $\mathrm{MPa}$ \\
Shear modulus & $\mathrm{G}$ & 66 & $\mathrm{MPa}$ \\
Density & $\rho$ & 925 & $\mathrm{Kg} / \mathrm{m}^{3}$ \\
\hline
\end{tabular}

\subsection{Failure due to yielding}

The membrane fails by yielding (or bursting) when the circumferential stresses, $\sigma_{\theta}$ in the membrane reach the yield stress of the material. The circumferential stress is considered for the failure criterion because, at maximum payload capability, the 
longitudinal stresses in the membrane tend to zero since the external forces acting on the robot are fully balanced by the internal pressure. The failure criterion for yielding is given by [21]:

$$
\sigma_{\theta}=\sigma_{y}
$$

where $\sigma_{y}$ denotes the yield stress of the material. We consider an eversion robot of radius, $R=2.5 \mathrm{~cm}$ and a length of $1 \mathrm{~m}$. Fig. 3(a) shows the circumferential stress in the robot body as a function of pressure. The black cross (x) in Fig. 3(a) shows the pressure at which the circumferential stress in the robot reaches the yield stress, indicating the yielding pressure. The corresponding force, $F_{\text {ext }}$ that would be exerted by the robot is given by $F_{\text {ext }}=\pi P R^{2}$ and is plotted in Fig. 3(b).

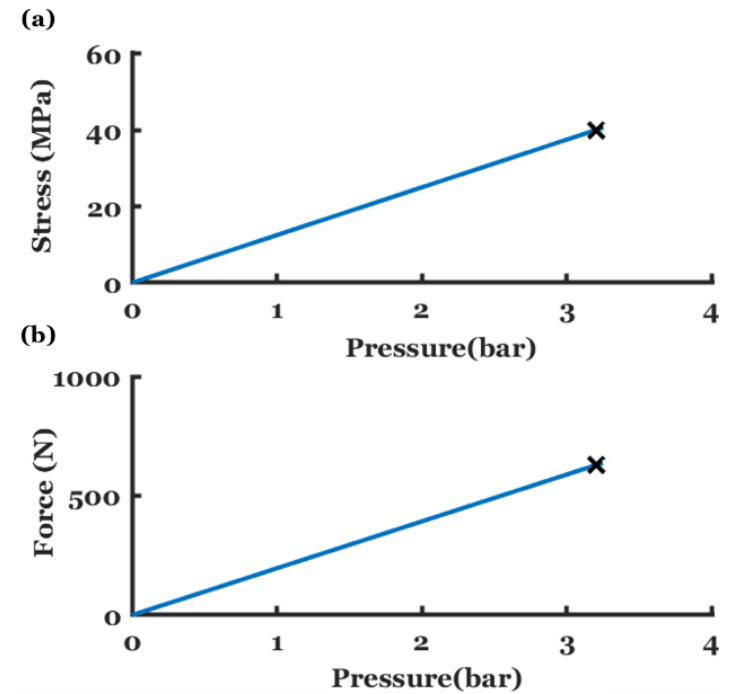

Fig. 3. (a) The circumferential stresses generated in the robot body upon inflation by internal pressure; (b) Blocked forces of the eversion robot as a function of internal pressure. The black crosses $(x)$ in both the plots indicate failure due to yielding.

\subsection{Failure due to buckling}

When the eversion robot is actuated against a load, compressive forces are generated on the robot. Due to the long lengths of eversion robots and the low radius to length ratio, the eversion robots are prone to buckling. An eversion robot buckled under compression when it encounters a rigid obstacle is shown in Fig. 4(a).

Under the assumptions that the cross-sections of the eversion robot along the length do not change, and in the limit of small strains, the critical load for buckling is given by [22]:

$$
F_{c r}=\frac{\left(E+\frac{P}{S}\right) I \Omega^{2}}{1+\Omega^{2} \frac{I}{S}+\Omega^{2} \frac{\left(E+\frac{P}{S}\right) I}{P+k G S}},
$$


where $\mathrm{S}$ denotes the cross-sectional area of the eversion robot, $I$ refers to the second moment of area about the central axis, $\Omega$ corresponds to the mode shape of the buckled beam given by $\Omega L=(2 n-1) \pi / 2$ and the primary mode of buckling is obtained by substituting $n=1$. The constant $k$ is the correction shear factor and is equal to 0.5 for circular tubes [23].

Eversion robots are generally made of very thin sheets or fabrics to facilitate the eversion mechanism, the effective shear modulus is generally low, and the effect of the pressure dominates the buckling behavior. When the internal pressure is zero and the shear modulus of material is low, the critical force for buckling recovers a value close to that of the Euler buckling load of a classical beam. However, the eversion robot cannot operate at zero pressure as this causes the longitudinal stresses in the membrane to disappear and even the smallest force will crush the robot. Fig. $4 \mathrm{~b}$ shows the critical buckling load at different internal pressures for an eversion robot of a length of $1 \mathrm{~m}$ and a radius of $2.5 \mathrm{~cm}$. The calculated buckling load at zero pressure is $1.328 \mathrm{~N}$.

(a)

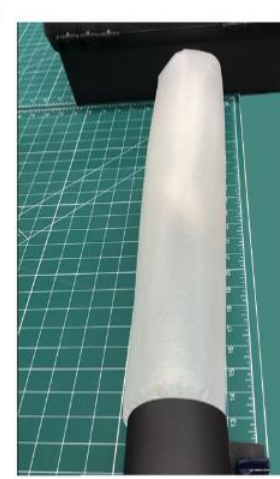

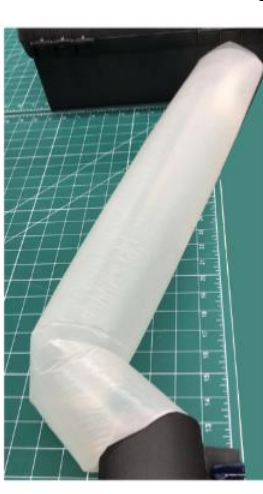

(b)

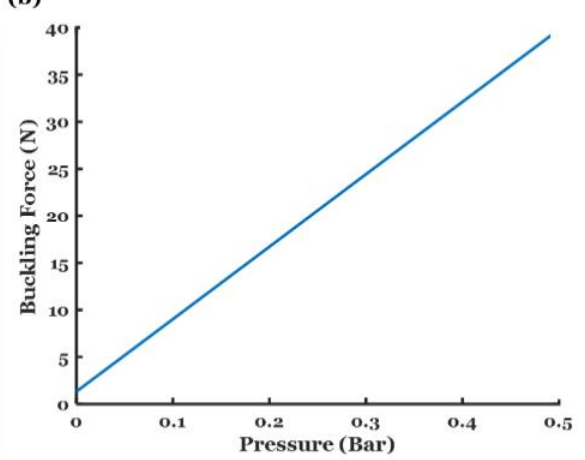

Fig. 4. (a) An eversion robot encounters a rigid obstacle and undergoes buckling; (b) Calculated critical buckling load for an eversion robot of radius $2.5 \mathrm{~cm}$ and length of $1 \mathrm{~m}$ at different internal pressure levels.

\subsection{Lateral collapse force}

The eversion robots may also be applied to carry loads in the lateral direction. For example, sensors mounted at the tip of a horizontally extending robot are subject to a lateral force due to gravity. A free body diagram of an eversion robot with a concentrated load applied laterally at its tip is shown in Fig. 5(a). Very long eversion robots may also experience large moments at the fixed end due to their own weight. These loads cause bending in the lateral direction. However, beyond a threshold, the eversion robot collapses with a kink in its surface as shown in Fig. 5(b).

Since the membrane making up an eversion robot is very thin, they cannot resist any compression and the longitudinal stress in all parts of the membrane along the length need to be non-negative. The condition for collapse of the eversion robot when a moment, $M$ is applied, is given by: 


$$
\sigma_{L}=\frac{M}{I} R>0
$$

For a concentrated force, $\mathrm{F}$ applied at the tip of the eversion robot inflated with a pressure $\mathrm{P}$ and balanced at equilibrium length, L by the tendon, Eqn. (9) can be evaluated to give the condition for lateral collapse of the robot as follows [24]:

$$
F_{\text {collapse }}=\frac{\pi R^{3} P}{2 L} \text {. }
$$

Fig. 5(c) shows the change in the critical force for lateral collapse of the eversion robot. The critical force at zero pressure is zero as expected and the lateral load bearing capacity increases with the increase in pressure.

(a)

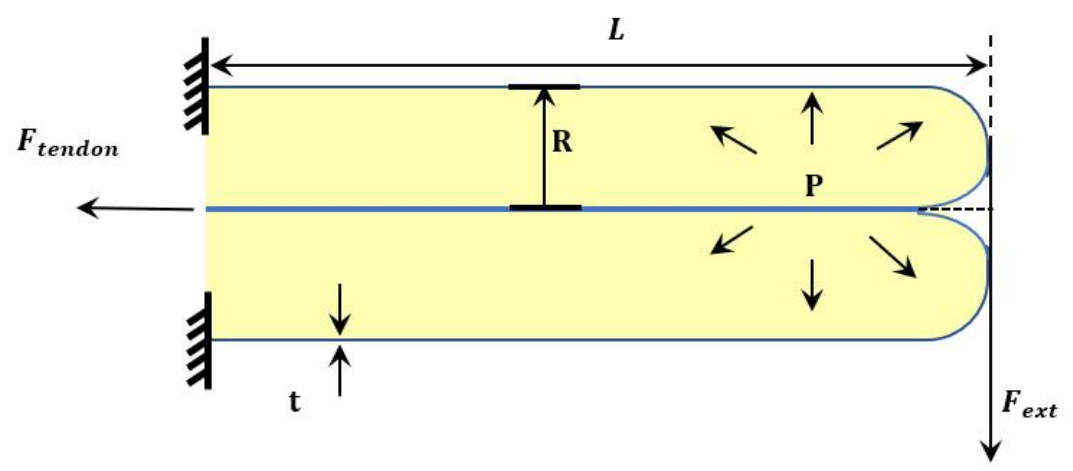

(b)

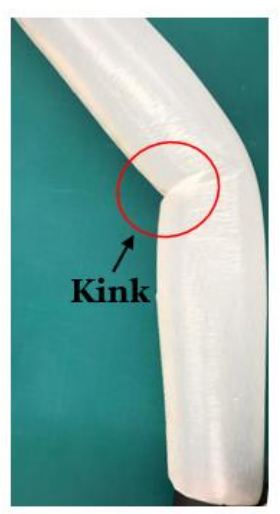

(c)

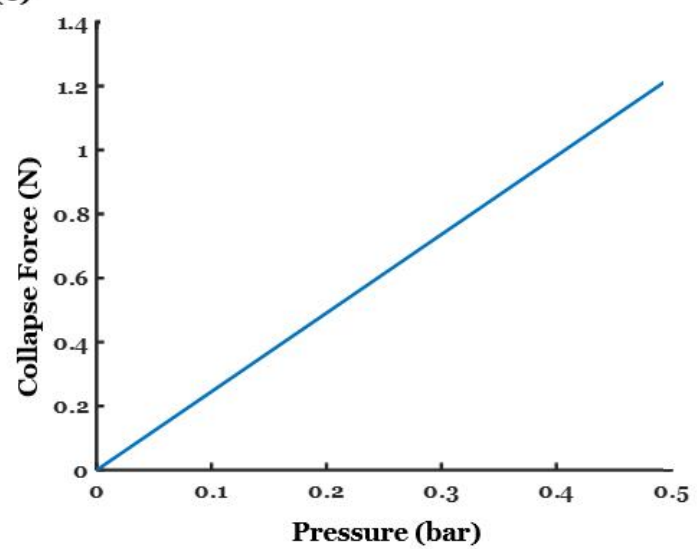

Fig. 5. (a) Free body diagram of an eversion robot with force applied laterally at the tip, (b) An eversion robot collapses laterally with a kink when the lateral force exceeds the critical limit; (c) Calculated critical buckling load for an eversion robot of radius $2.5 \mathrm{~cm}$ and length of $1 \mathrm{~m}$ at different internal pressures. 


\subsection{Operational limits}

The geometrical parameters of the eversion robot play an important role in determining the payload capabilities and the mode of failure encountered by the robot when loaded axially and laterally. Since eversion robots grow in length during their operation, the mechanical design should keep in mind the evolution of operation limits with changes in the everted length. To aid in this procedure, we calculate the maximum obtainable payloads in the axial and lateral directions before the robot encounters yielding, buckling or lateral collapse.

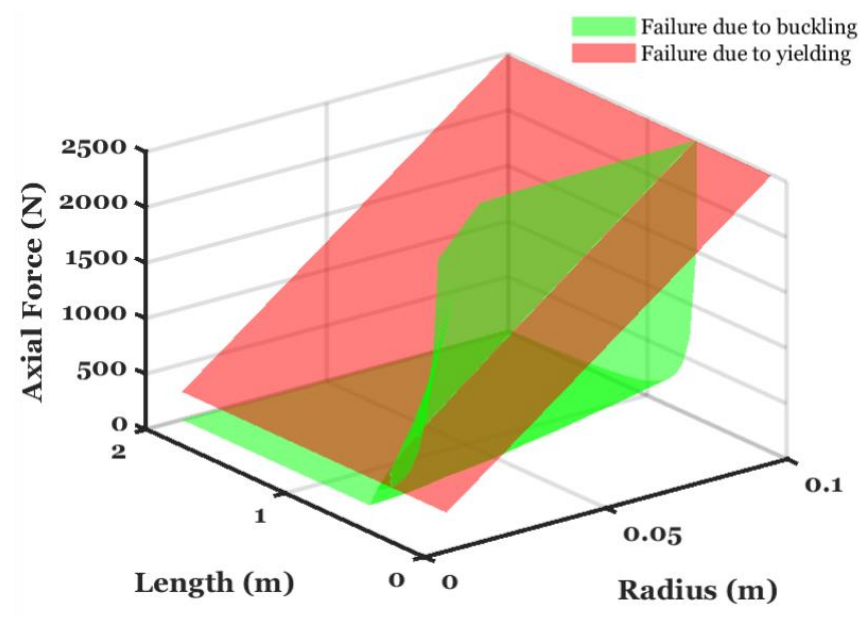

Fig. 6. Operational limits of eversion robots with different radii and everted lengths. The pink and green surfaces show the maximum axial force considering yielding and buckling as the failure criterion respectively.

Fig. 6 shows a 3D map of the maximum axial payload capability for different combinations of radius and everted lengths. The pressure for the critical points has been abstracted and only the maximum achievable forces for the combinations of radii and everted lengths have been plotted. The pink surface shows the maximum force considering yielding as the failure criterion and the green surface shows the maximum load at which buckling occurs for the given combination of radius and everted length. The plot for failure due to buckling shows a very nonlinear pattern and cannot be obtained as a closed form solution. This is because both the axial force that can be generated by the robot and the critical force for buckling vary with pressure. When the axial force generated by the robot becomes equal to the critical load for buckling for the given pressure, the robot undergoes buckling.

As the radius of the eversion robot is increased, the payload capability always increases for the same material at a given everted length of the robot. The dynamic variation of payload capability with the change in length of the eversion robot can also be noted from the figure. When the radius of the robot is large, the maximum force does not vary with the length and is dependent only on the yield strength of the material that 
the robot is composed of. However, beyond a particular length, the robot becomes prone to buckling and the payload capability rapidly decreases. The maximum achievable axial payload at an everted length of $2 \mathrm{~m}$ for a radius of $5 \mathrm{~cm}$ is $2.95 \mathrm{~N}$ as compared to $23.34 \mathrm{~N}$ achievable for a radius of $10 \mathrm{~cm}$.

The distribution of the lateral payloads before collapse for different radii and everted lengths of the robot is shown in Fig. 7. From Eqn. (10), we see that the lateral payload linearly increases with the internal pressure. It is evident that the maximum lateral payload would be attained at the maximum possible internal pressure which occurs at the yield limit of the robot. From Eqns. (6-7), we get the maximum pressure at yield as, $P_{\max }=2 t \sigma_{y} / R$. This internal pressure can be achieved at any everted length by applying force to the tendon. However, within the limit of small deformations, we assume that the tendon does not affect the lateral load bearing capability as it remains in the neutral plane of the eversion robot. By substituting $P_{\text {max }}$ in Eqn. (10), the maximum possible lateral force is given by:

$$
F_{\text {lateral }}=\frac{\pi R^{2} \sigma_{y} t}{L} .
$$

Eqn. (11) is evaluated for different radii and everted lengths and shown as a 3D surface in Fig. 7. The maximum achievable lateral payload at an everted length of $2 \mathrm{~m}$ for a radius of $5 \mathrm{~cm}$ is $15.71 \mathrm{~N}$ as compared to $62.83 \mathrm{~N}$ achievable for a radius of $10 \mathrm{~cm}$. We observe from Figs. 6 and 7 that the critical load for axial buckling is more sensitive to changes in length and reduces at a faster rate as compared to the critical load for lateral collapse.

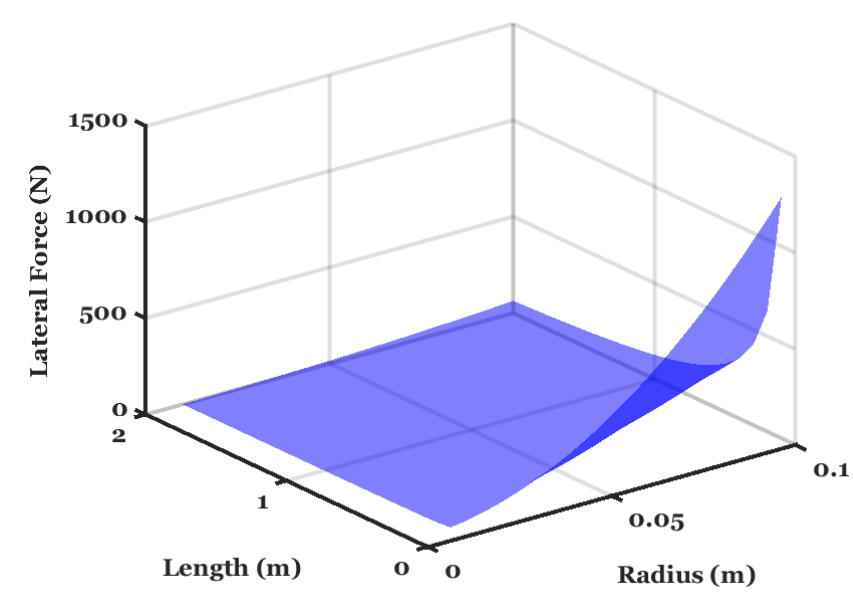

Fig. 7. Operational limits of eversion robots with different radii and everted lengths. The blue surface shows the maximum lateral force beyond which the robot collapses. 


\section{Conclusions}

In this paper, we present an analysis to determine the payload capabilities of eversion robots. We have investigated the effects of the radius of the robot and the everted length on the axial and lateral payload bearing capabilities of the robots. We studied yielding, buckling due to compression and collapse due to lateral loading as the main modes of failure. The results show an interplay of these failure modes and suggest that the mechanics and the intended actuation ranges should be kept in mind to optimize the payload of the eversion robots. The analysis presented in this paper can aid in designing eversion robots with different payload capabilities and lays the foundation for high payload eversion robots.

\section{Acknowledgements}

This work was supported in part by the EPSRC National Centre for Nuclear Robotics project (EP/R02572X/1), and the Innovate UK project WormBot (104059).

\section{References}

1. Shintake, J., Rosset, S., Schubert, B., Floreano, D., Shea, H.: Versatile Soft Grippers with Intrinsic Electroadhesion Based on Multifunctional Polymer Actuators. Adv. Mater. 28, 231-8 (2015).

2. Godaba, H., Li, J., Wang, Y., Zhu, J.: A Soft Jellyfish Robot Driven by a Dielectric Elastomer Actuator. IEEE Robot. Autom. Lett. 1, 624-631 (2016).

3. Behl, M., Kratz, K., Noechel, U., Sauter, T., Lendlein, A.: Temperature-memory polymer actuators. Proc. Natl. Acad. Sci. 110, 12555-12559 (2013).

4. Liu, Z., Calvert, P.: Multilayer hydrogels as muscle-like actuators. Adv. Mater. 12, 288291 (2000).

5. Althoefer, K.: Antagonistic actuation and stiffness control in soft inflatable robots. Nat. Rev. Mater. 3, 76 (2018).

6. Shepherd, R.F., Ilievski, F., Choi, W., Morin, S.A., Stokes, A.A., Mazzeo, A.D., Chen, X., Wang, M., Whitesides, G.M.: Multigait soft robot. Proc. Natl. Acad. Sci. 108, 20400-20403 (2011).

7. Marchese, A.D., Katzschmann, R.K., Rus, D.: A recipe for soft fluidic elastomer robots. Soft Robot. 2, 7-25 (2015).

8. Niiyama, R., Rus, D., Kim, S.: Pouch motors: Printable/inflatable soft actuators for robotics. In: Robotics and Automation (ICRA), 2014 IEEE International Conference on. pp. 6332-6337. IEEE (2014).

9. Liang, X., Cheong, H., Sun, Y., Guo, J., Chui, C.K., Yeow, C.-H.: Design, Characterization, and Implementation of a Two-DOF Fabric-Based Soft Robotic Arm. IEEE Robot. Autom. Lett. 3, 2702-2709 (2018).

10. Li, J., Godaba, H., Zhang, Z.Q., Foo, C.C., Zhu, J.: A soft active origami robot. Extrem. Mech. Lett. 24, 30-37 (2018). 
11. Abrar, T., Putzu, F., Althoefer, K.: Soft Wearable Glove for Tele-Rehabilitation Therapy of Clenched Hand/Fingers Patients. In: Workshop on Computer/Robot Assisted Surgery. , London (2018).

12. Hawkes, E.W., Blumenschein, L.H., Greer, J.D., Okamura, A.M.: A soft robot that navigates its environment through growth. Sci. Robot. 2, eaan3028 (2017).

13. Blumenschein, L.H., Gan, L.T., Fan, J.A., Okamura, A.M., Hawkes, E.W.: A TipExtending Soft Robot Enables Reconfigurable and Deployable Antennas. IEEE Robot. Autom. Lett. 3, 949-956 (2018).

14. Naclerio, N.D., Hubicki, C.M., Aydin, Y.O., Goldman, D.I., Hawkes, E.W.: Soft Robotic Burrowing Device with Tip-Extension and Granular Fluidization. In: 2018 IEEE/RSJ International Conference on Intelligent Robots and Systems (IROS). pp. 5918-5923. IEEE (2018).

15. Putzu, F., Abrar, T., Althoefer, K.: Plant-Inspired Soft Pneumatic Eversion Robot. In: 2018 7th IEEE International Conference on Biomedical Robotics and Biomechatronics (Biorob). pp. 1327-1332. IEEE (2018).

16. Althoefer, K.A.: Neuro-fuzzy motion planning for robotic manipulators, (1997).

17. Lockhart, J.A.: An analysis of irreversible plant cell elongation. J. Theor. Biol. 8, 264275 (1965)

18. Blumenschein, L.H., Okamura, A.M., Hawkes, E.W.: Modeling of bioinspired apical extension in a soft robot. In: Conference on Biomimetic and Biohybrid Systems. pp. 522-531. Springer (2017).

19. Timoshenko, S.P., Gere, J.M.: Theory of elastic stability, (1961).

20. Jordan, J.L., Casem, D.T., Bradley, J.M., Dwivedi, A.K., Brown, E.N., Jordan, C.W.: Mechanical properties of low density polyethylene. J. Dyn. Behav. Mater. 2, 411-420 (2016).

21. Timoshenko, S.: Strength of materials Part 1. D. Van Nostrand Co., Inc (1940).

22. Le Van, A., Wielgosz, C.: Bending and buckling of inflatable beams: some new theoretical results. Thin-walled Struct. 43, 1166-1187 (2005).

23. Cowper, G.R.: The shear coefficient in Timoshenko's beam theory. J. Appl. Mech. 33, 335-340 (1966).

24. Comer, R.L., Levy, S.: Deflections of an inflated circular-cylindrical cantilever beam. AIAA J. 1, 1652-1655 (1963). 